\title{
Decontamination of Creutzfeldt - Jakob Disease and other transmissible agents
}

\author{
Laura Manuelidis \\ Yale Medical School, Section of Neuropathology, 310 Cedar Street, New Haven, Connecticut 06510, USA
}

\begin{abstract}
The bovine spongiform encephalopathy (BSE) epidemic in cows, and the recent BSE-linked human infections, present new public health problems. More rigorous measures are needed to prevent additional transmissions. Tissue from established but undiagnosed human infections can contaminate medical supplies and instruments. We tested guanidine thiocyanate (GdnSCN) solutions and found them to be highly effective in disrupting the infectious agent, even in very complex tissues such as whole brain. It may be prudent now to use this reagent routinely in surgical and other relevant settings.
\end{abstract}

Keywords: scrapie; bovine spongiform encephalopathy; hepatitis B; prion

Creutzfeldt-Jakob Disease (CJD) is a rare but devastating neurodegenerative disease. Although caused by a transmissible agent, clinical symptoms may not become apparent for as long as 30 years after infection. Because white blood cells carry the infectious agent, in 1978 we considered the possibility that this disease could be transmitted through routine medical and surgical procedures (Manuelidis et al, 1978). Over the past 10 years England has witnessed an explosive epidemic infection of cows with a similar agent that induces bovine spongiform encephalopathy (BSE). The BSE agent probably originated in processed feed contaminated with the sheep scrapie agent (Anderson et al, 1996) but it is biologically distinct from common scrapie agent strains in sheep (Bruce, 1993). I suggested that variants of the scrapie agent selected in cows might become more virulent for humans than was commonly assumed (Manuelidis, 1994). Whereas endemic scrapie infections of sheep have not been linked to human disease, several unusual human CJD cases have now been linked to the bovine infection (Will et al, 1996; Lasemezas et al, 1996). PrP polymorphisms are sometimes considered critical for infection. However, the single PrP 129 Met-Met polymorphism found in these patients is very common (38\% of Caucasians), and it is unlikely that this polymorphism is always present in the wide variety of animals that have

Correspondence: Laura Manuelidis

Received 21 November 1996; accepted 27 November 1996 acquired BSE. There is the unsettling possibility that the incidence of CJD may rise significantly because many individuals, including young people, have been repeatedly exposed to this new and apparently more virulent agent strain.

There is currently no in vivo test to detect transmissible encephalopathies during early stages of infection in any species. Nor are there any molecular markers to distinguish agent strains that are more or less virulent for humans. Inadvertent transmissions are therefore problematic. Of special concern is the likelihood of enhanced virulence of the human adapted BSE agent. Many experiments have shown that once these agents are passaged in a new host, they typically become more virulent for that host, yielding more rapidly progressive disease. Thus the spread of infection from already identified BSE-infected people, as well as from other asymptomatic infected individuals, must be considered. Sensitive tests to detect early infections and prevent iatrogenic spread of disease will ultimately rely on the molecular characteristics of the infectious agent. However, these are not resolved, and there are two fundamentally different concepts in play. One predominant notion is that a host protein, PrP, converts itself into an infectious protein or prion (Prusiner, 1991). The alternate view is that the agent is a virus that affects host PrP (Manuelidis, 1994; Bruce and Dickinson, 1987; Diringer et al, 1991; Manuelidis and Fritch, 1996). Neither view is experimentally proven. Although PrP is clearly involved in pathogenesis, purified PrP does not correlate with titer and has failed to transmit infectious disease. On the other hand, although 
disruption of viral particles reduces CJD titer (Manuelidis et al, 1978), only candidate viral sequences have been defined (Dron and Manuelidis, 1996).

Immediate health related initiatives must therefore rest on proven experimental approaches to reduce titer and thereby minimize the risk of contracting disease. One practical problem is decontamination of human and animal tissues. Because the agent is infectious at high dilutions, is carried in the reticuloendothelial system, and is highly concentrated in the brain at asymptomatic stages of disease (Xi et al, 1992; Manuelidis and Fritch, 1996), general and simple methods to significantly reduce tissue infectivity are needed. These methods should be applicable for routine use on all residual tissues because infectivity cannot be predicted. In a surgical setting, tissue fragments can adhere to operative instruments. Some larger instruments, such as dental drill holders, are not disposable. Even though these instruments can be at some distance from the operative field, they are exposed to tissue microdroplets. Dental work also frequently exposes nerve roots, and thus dental procedures could become a common source for the spread of CJD. Additionally, the expense of discarding specialized surgical instruments after a single use may not be possible in many countries. Finally, previous and proposed new slaughter of cattle to reduce BSE exposure in Europe, and the routine removal of brain and spinal cord during butchering, can lead to contamination of large facilities.

Up to the present time three methods have been used to reduce instrument contamination and these generally reduce titer by $>3$ logs. They are (i) exposure to household bleach (a procedure that can corrode many fine instruments, mechanical parts and stainless steel, (ii) prolonged autoclaving (a method not feasible for many large instruments or surfaces) and (iii) immersion in concentrated alkaline solutions, i.e., $0.1-1 \mathrm{M} \mathrm{NaOH}$. The latter solutions are corrosive not only to instruments and surfaces, but also cause severe skin burns.

In studies to uncover essential molecular components of the CJD agent we found that concentrations of $\mathrm{GdnHCl}$ as low as $2.5 \mathrm{M}$ effectively disrupted the nucleic acids and capsid proteins of viral particles. Concomitantly, CJD titer was reduced by $\sim 3$ logs $(99.7 \%)$. In contrast, other reagents that preserved viral particles while removing PrP maintained high levels of infectivity (Manuelidis et al, 1995). We therefore suggested that GdnHCl treatments could be of value in reducing contamination and started experiments to validate this approach. Others subsequently hypothesized that GdnHCl might be useful for dental instrument decontamination (Oken and McGeer, 1995). We presumed that more disruptive GdnSCN solutions could result in even greater levels of agent inactivation and therefore verified this experimentally.
To simplify the method for clinical settings, a commercially available solution of $4 \mathrm{M}$ GdnSCN was used (RNA lysis buffer \#40082, Perkin Elmer). This solution has a similar composition as the one we use to efficiently extract nucleic acids from more purified CJD preparations (Dron and Manuelidis, 1996). It conveniently contains a detergent that can aid in tissue penetration and disruption, and also has a reasonable shelf life of $>1$ year. Moreover, this solution can be easily modified to further enhance decontamination. Moderate heat treatment was also tested to find if agent inactivation could be augmented. The temperatures tested are easily achieved with old instrument sterilizers in dentists offices. Brain tissue from our long established CJD hamster model (serial passage 31 , strain SY) was chosen because it yields reproducible titers. The very high lipid composition and complexity of brain can compromise inactivating procedures and therefore whole brain was chosen as a difficult inactivation target. Experimental groups of six hamsters each were injected with: (1) CJD homogenates in saline without inactivation; (2) CJD saline homogenates treated at $75^{\circ} \mathrm{C}$ for $25 \mathrm{~min}$; (3) CJD homogenates lysed in GdnSCN and (4) CJD GdnSCN homogenates treated at $75^{\circ} \mathrm{C}$ for $25 \mathrm{~min}$.

To maximize viral input each half of a CJD infected brain was directly homogenized in either normal saline or GdnSCN lysis buffer (10\% w/v). A $100 \mathrm{ml}$ aliquot of each respective homogenate was heated in a $1.5 \mathrm{ml}$ polypropylene tube in a PCR machine while the parallel homogenates were kept at $22^{\circ} \mathrm{C}$. Each of the four homogenates were then diluted to $0.5 \mathrm{mg}$ brain $/ \mathrm{ml}$ and $50 \mu \mathrm{l}$ was inoculated intracerebrally per hamster (Manuelidis et al, 1995). The large dilution was calculated (1) to preclude any toxic effects of GdnSCN, (2) to yield incubation time titers in the linear range for saline homogenates (Manuelidis et al, 1987) and (3) to approximate end-point $\mathrm{LD}_{50}$ determinations for GdnSCN samples.

As summarized in Table 1, hamsters inoculated with CJD brain in saline developed clinical disease by 166 days. There was a small ( $\sim 3$-fold or 0.55 logs) but statistically significant decrease in titer with moderate heating in saline. Typical CJD changes (Manuelidis and Fritch, 1996) were verified histologically in both saline groups (data not shown). A far more substantial reduction in titer was found in GdnSCN treated parallel samples. Indeed, none of these animals has yet shown any signs of disease at $>350$ days post inoculation. With GdnSCN the titer was reduced by $\sim 5$ logs $\left(2.3 \times 10^{8} / \mathrm{gm}\right.$ to $\left.<10^{3} / \mathrm{gm}\right)$. Because the GdnSCN treatment alone was so effective, we were unable to determine if heating GdnSCN homogenates increased inactivation. Nonetheless, the saline controls indicate that heating at $75^{\circ} \mathrm{C}$ or higher should yield even more effective inactivations, although toxic fumes might be released at $75^{\circ} \mathrm{C}$. 
Table 1

\begin{tabular}{lcc}
\hline Group & Days signs (s.e.m.) & Titer $/ g m$ (s.e.m.) \\
\hline (1) CJD-saline & $166.2(1)$ & $2.3 \times 10^{8}\left(2.1 \times 10^{7}\right)$ \\
(2) CJD-saline and & $180.2(0.5)$ & $6.5 \times 10^{7}\left(2.8 \times 10^{6}\right)$ \\
heat & $>350$ & $\leqslant 10^{3}$ \\
(3) CJD-GdnSCN & $>350$ & $\leqslant 10^{3}$ \\
(4) CJD-GdnSCN & & \\
and heat & &
\end{tabular}

Days incubation to clinical signs of disease and titer per gram of brain for each experimental group. Two-tailed t-test showed significant reduction in homogenate titer after moderate heating in saline $\left(P<10^{-6}\right)$, with far more inactivation after GdnSCN treatment (longer incubation time in groups 3 and 4). Titer calculations are based on the conservative estimate that 1 infectious unit (IU) will produce clinical signs at 294 days, and thus end-point determinations at 600 days yield a fraction of 1 $\mathrm{IU}$, eg one of five animals with disease at 600 days is equivalent to $0.2 \mathrm{IU}$ in the inoculum

Additional experiments on infectious concentrates have shown heating at $55^{\circ} \mathrm{C}$ further enhances disruption by molecular analyses, and heat increases penetration into mechanical lubricants (Lewis and Arens, 1995). Exposure of stainless steel surgical instruments (Dumont tweezers) for $24 \mathrm{~h}$ to $4 \mathrm{M}$ GdnSCN, moreover, showed no corrosive effects. Therefore nondisposable and fine surgical instruments may be significantly decontaminated by immersion and sonication in the above GdnSCN solution (GdnSCN wipes for steel surfaces can be incinerated). These treatments may have universal application for the inactivation of many other infectious agents, including non-enveloped viruses that are relatively resistant to inactivation such as Hepatitis B. A recent outbreak of Hepatitis B was traced to incompletely sterilized EEG needles (see below) and perhaps could have been avoided with the above precautions.

The outlined protocol is also based on our inability to find any reconstitution of CJD infectivity when more purified preparations were treated with less concentrated and disruptive $\mathrm{GdnHCl}$ solutions (Manuelidis et al, 1995). After dilution of GdnHCl, the reduction in titer remained. Thus washing trace GdnSCN should have few risks. Using similar GdnHCl treatment and dilution, another laboratory reported that $\mathrm{PrP}$ rapidly refolded into an 'infectious conformation', as assessed by gel assays. However, there was no data showing infectious titer was restored (Bessen et al, 1995). Moreover, similar refolding experiments have been irreproducible, with PrP conversion ascribed to an artifact of incompletely denatured aggregates (Riesner et al, 1996). Older studies have likewise shown reductions in scrapie titer with other Gdn solutions but the application of such treatments for decontami- nating instruments and surfaces have not been previously considered.

Use of a standard solution and protocol should help eliminate variable inactivations. Although somewhat different sensitivities to the same chemical and physical treatments have been reported with different agent strains (eg, hamster adapted scrapie and CJD), all preparations are highly sensitive to >3 M GdnSCN. Additionally, minor methodological and preparation differences can often account for vastly different claims about the resistance of these agents. The sometimes magical qualities ascribed to these agents should be viewed in the framework of conventional viral studies where absolute inactivations are rarely achieved. Indeed, glutaraldehyde treated HIV material can be infectious (Lewis and Arens, 1995), and after dry heat treatment of tissue products at $80^{\circ} \mathrm{C}$, the risk of Hepatitis C infections can still be as high as $10 \%$ (Rizza et al, 1993). These two enveloped viruses are relatively sensitive in the viral spectrum. Furthermore, Hepatitis B, a more resistant virus, was not inactivated when dry heat sterilizers were used to decontaminate reusable subcutaneous EEG electrodes (McKinnon, 1996).

Incomplete inactivations may be due, at least in part, to inadequate tissue penetration. Penetration should be significantly enhanced with strongly disruptive GdnSCN. Nonetheless, the above recommendations are made with the recognition that absolute inactivations of CJD-like agents or other resistant viruses cannot yet be guaranteed. Indeed, it is possible that the incomplete but still harsh rendering process used in the production of scrapie infected feed itself selected for more resistant agent variants causing BSE. Large BSE infected cattle are currently being sterilized by cremation, and some cow remains have already been dumped in open pits in northern Scotland. There is the possibility that some remains may be incompletely ashed, and it may be prudent now to take added new steps to prevent the spread of residual, and perhaps more resistant agent variants to wild animals. Treatment of incompletely ashed or partially inactivated remains with GdnSCN derivatives could help prevent further propagation of evolving virulent strains.

\section{Acknowledgements}

I thank William Fritch for excellent technical assistance. This work was supported by NIH grants NS12674 \& NS34569. 


\section{References}

Anderson RM, Donnelly CA, Ferguson NM, Woolhouse MEJ, Watt CJ, Udy HJ, MaWhinney S, Dunstan SP, Southwood TRE, Wilesmith JW (1996). Transmission dynamics and epidemiology of BSE in British cattle. Nature 382: 779-788.

Bessen RA, Kocisko DA, Raymond GH, Nandan S, Lansbury PT, Caughey B (1995). Non-genetic propagation of strain-specific properties of scrapie prion protein. Nature 375: 698-700.

Bruce ME, Dickinson AG (1987). Biological evidence that scrapie has an independent genome. J Gen Virol 68: $79-89$.

Bruce ME (1993). Scrapie strain variation and mutation. Br Med Bull 49: 822-838.

Diringer H, Blode H, Oberdieck U (1991). Virus-induced amyloidosis in scrapie involves a change in covalent linkage in the preamyloid. Arch Virol 118: 127-131.

Dron M, Manuelidis L (1996). Visualization of viral candidate cDNAs in infectious brain fractions from Creutzfeldt-Jakob Disease by representational difference analysis. J Neurovirol 2: 240-248.

Lasemezas C, Deslys J-P, Demaimay R, Adjou K, Lamoury F, Dormont D, Robain O, Ironside J, Hauw J-J (1996). BSE transmission to macaques. Nature 381: $733-734$.

Lewis DL, Arens M (1995). Resistance of microorganisms to disinfection in dental and medical devices. Nature Medicine 1: 956-958.

Manuelidis EE, Gorgacz EJ, Manuelidis L (1978). Viremia in experimental Creutzfeldt-Jakob disease. Science 200: $1069-1071$.

Manuelidis L, Sklaviadis T, Manuelidis EE (1987). Evidence suggesting that $\operatorname{PrP}$ is not the infectious agent in Creutzfeldt-Jakob disease. $E M B O J$ 6: $341-$ 347.
Manuelidis L (1994). The dimensions of CreutzfeldtJakob Disease. Transfusion 34: 915-928.

Manuelidis L, Sklaviadis T, Akowitz A, Fritch W (1995). Viral particles are required for infection in neurodegenerative Creutzfeld-Jakob Disease. Proc Natl Acad Sci USA 92: 5124-5128.

Manuelidis L, Fritch W (1996). Infectivity and host responses in Creutzfeldt-Jakob Disease. Virology 215: $46-59$.

McKinnon DJ (1996). Thirty new cases of Hepatitis B found. All had EEGs at six clinics, officials say. Toronto Star page SC4.

Oken R, McGeer P (1995). Letter to the editor. J Dental Res 74: 1836.

Prusiner SB (1991). Molecular biology of prion diseases. Science 252: 1515-1522.

Riesner D, Kellings K, Wiese U, Wulfert M, Mirenda C, Prusiner SB (1996). Disruption of prion rods generates 10-nm spherical particles having high a-helical content and lacking scrapie infectivity. J Virol 70: $1714-1722$.

Rizza CR, Fletcher ML, Kernoff PB (1983). Confirmation of viral safety of dry heated factor VIII concentrate (8Y) prepared by Bio Products Laboratory (BPL): a report on behalf of UK Haemophilia Centre Directors. Br J Haematol 84: 269-272.

Will R, Ironside J, Zeidler M, Cousens S, Estibeiro K, Alperovitch A, Poser S, Pocchiari M, Hofman A, Smith P (1996). A new variant of Creutzfeldt-Jakob Disease in the UK. Lancet 347: 921-925.

Xi YG, Ingrosso A, Ladogana A, Masullo C, Pocchiari M (1992). Amphotericin B treatment dissociates in vivo replication of the scrapie agent from PrP accumulation. Nature 356: 598-601. 